\title{
Application of Successive Linearisation Method to Squeezing Flow with Bifurcation
}

\author{
S. S. Motsa, ${ }^{1}$ O. D. Makinde, ${ }^{2}$ and S. Shateyi ${ }^{3}$ \\ ${ }^{1}$ School of Mathematical Sciences, University of KwaZulu-Natal, Private Bag X01, Scottsville, Pietermaritzburg 3209, South Africa \\ ${ }^{2}$ Faculty of Military Science, Stellenbosch University, Private Bag X2, Saldanha 7395, South Africa \\ ${ }^{3}$ Department of Mathematics \& Applied Mathematics, University of Venda, Private Bag X5050, Thohoyandou 0950, South Africa
}

Correspondence should be addressed to S. S. Motsa; sandilemotsa@gmail.com

Received 28 September 2013; Accepted 16 December 2013; Published 2 January 2014

Academic Editor: R. N. Jana

Copyright (C) 2014 S. S. Motsa et al. This is an open access article distributed under the Creative Commons Attribution License, which permits unrestricted use, distribution, and reproduction in any medium, provided the original work is properly cited.

\begin{abstract}
This paper employs the computational approach known as successive linearization method (SLM) to tackle a fourth order nonlinear differential equation modelling the transient flow of an incompressible viscous fluid between two parallel plates produced by a simple wall motion. Numerical and graphical results obtained show excellent agreement with the earlier results reported in the literature. We obtain solution branches as well as a turning point in the flow field accurately. A comparison with numerical results generated using the inbuilt MATLAB boundary value solver, bvp4c, demonstrates that the SLM approach is a very efficient technique for tackling highly nonlinear differential equations of the type discussed in this paper.
\end{abstract}

\section{Introduction}

Studies related to transient flows produced by a simple wall motion have been of interest for several years due to its practical importance in understanding several engineering and physiological flow problems. For instance, the entire conduits in human body are flexible and also collapsible. That is, when the external pressure exceeds the internal pressure, the crosssectional area can be significantly reduced, if not fully diminished. The cross-section may eventually return to its original shape when the external pressure is reduced, and, consequently, normal internal fluid flow can be restored [1]. Other applications can be found in unsteady loading, which is met frequently in many hydrodynamical machines and apparatus [2]. In the light of these applications, squeezing flow in a channel has been studied by many authors; mention may be made of research studies [3-6]. This problem admits similarity variable $[7,8]$, thereby reducing the unsteady Navier-Stokes equations to a parameter dependent fourth order nonlinear ordinary differential equation for the similarity function.

Generally speaking, nonlinear problems and their solutions provide an insight into inherently complex physical process in the system. The nonlinear nature of the model equations in most cases precludes its exact solution. Several approximation techniques have been developed to tackle this problem such as the homotopy analysis method [9-11], homotopy perturbation method [12,13], spectral homotopy analysis method $[14,15]$, and variational iteration method [16]. In this paper, we employ the successive linearisation method [17-19] to tackle a fourth order nonlinear boundary value problem that governs the squeezing flow problem between parallel plates. In this work, we assess the applicability of the SLM approach in solving nonlinear problems with bifurcations. Such problems are very difficult to resolve numerically near the bifurcation point. Numerical and graphical results obtained using the new SLM approach are validated through comparison with numerical results generated using the inbuilt MATLAB boundary value solver, bvp4c, for different values of the governing physical parameters. In following sections, the problem is formulated, analysed, and discussed.

\section{Mathematical Formulation}

Consider a transient flow of an incompressible viscous fluid between parallel plates driven by the normal motion of 


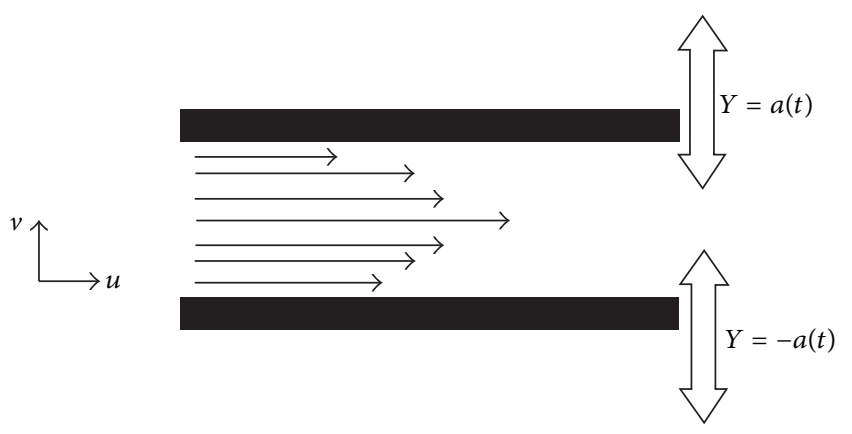

FIGURE 1: Schematic diagram of the problem.

the plates. Take a Cartesian coordinate system $(x, y)$ where $x$ lies in the streamwise direction and $y$ is the distance measured in the transverse direction. Let $u$ and $v$ be the velocity components in the directions of $x$ and $y$ increasing, respectively. It is assumed that the two plates are at $y=$ $\pm a_{0} \sqrt{(1-\alpha t)}$, where $a_{0}$ is the position at time $t=0$ as shown in Figure 1.

When $\alpha$ is positive, the two plates are squeezed symmetrically until they touch at $t=1 / \alpha$. Negative values of $\alpha$ represent the symmetrical separation of the plates. The length of the plates is assumed to be much larger than the gap width at any time such that the end effects could be neglected. Following [4, 6-8], the two-dimensional governing equation of motion in terms of vorticity $(\omega)$ and stream function $(\Psi)$ formulation is given as

$$
\frac{\partial \omega}{\partial t}+\frac{\partial(\omega, \Psi)}{\partial(x, y)}=\nu \nabla \omega, \quad \omega=-\nabla^{2} \Psi,
$$

with

$$
\begin{gathered}
\frac{\partial \Psi}{\partial y}=0, \quad \frac{\partial \Psi}{\partial x}=-\frac{d a}{d t}, \quad \text { on } y=a(t), \\
\frac{\partial^{2} \Psi}{\partial y^{2}}=0, \quad \frac{\partial \Psi}{\partial x}=0, \quad \text { on } y=0 .
\end{gathered}
$$

We introduce the following transformations:

$$
\begin{gathered}
\eta=\frac{y}{a_{0} \sqrt{1-\alpha t}}, \quad \Psi=\frac{\alpha a_{0} x F(\eta)}{2 \sqrt{(1-\alpha t)}}, \\
\omega=-\frac{\alpha x}{2 a_{0}(\sqrt{(1-\alpha t)})^{3}} \frac{d^{2} F}{d \eta^{2}} .
\end{gathered}
$$

Substituting (3) into (1) and (2), we obtain

$$
\begin{gathered}
\frac{d^{4} F}{d \eta^{4}}=R\left(\frac{d^{2} F}{d \eta^{2}} \frac{d F}{d \eta}-F \frac{d^{3} F}{d \eta^{3}}+\eta \frac{d^{3} F}{d \eta^{3}}+3 \frac{d^{2} F}{d \eta^{2}}\right), \\
\frac{d F}{d \eta}=0, \quad F=1, \text { on } \eta=1, \\
\frac{d^{2} F}{d \eta^{2}}=0, \quad F=0, \text { on } \eta=0,
\end{gathered}
$$

where $R=a_{0}^{2} \alpha / 2 \nu$ is the local Reynolds number $(R>0$ represents squeezing and $R<0$ represents separation). The wall skin friction is given by

$$
\tau_{w}=-\mu \frac{\partial u}{\partial y}=-\frac{\alpha \mu x}{2 a_{0}(\sqrt{(1-\alpha t)})^{3}} \frac{d^{2} F}{d \eta^{2}}, \quad \text { at } \eta=1
$$

where $\mu$ is the dynamic coefficient of viscosity. From the axial component of the Navier-Stokes equations, the pressure drop in the longitudinal direction can be obtained. Let

$$
\frac{\partial P}{\partial x}=\frac{\mu \alpha x A}{2 a_{0}^{2}(1-\alpha t)^{2}}
$$

and we obtain

$$
A=\frac{d^{3} F}{d \eta^{3}}-R\left[\left(\frac{d F}{d \eta}\right)^{2}-F \frac{d^{2} F}{d \eta^{2}}+\eta \frac{d^{2} F}{d \eta^{2}}+2 \frac{d F}{d \eta}\right] .
$$

In the following section, (4)-(6) will be solved using successive linearization method and other important flow properties like the skin friction and pressure drop will be determined.

\section{Successive Linearisation Method (SLM) Approach}

The proposed linearisation method of solution, hereinafter referred to as the successive linearisation method (SLM), is based on the assumption that the unknown function $F(\eta)$ can be expanded as

$$
F(\eta)=F_{i}(\eta)+\sum_{m=0}^{i-1} F_{m}(\eta), \quad i=1,2,3, \ldots
$$

where $F_{i}$ are unknown functions. The solutions of $F_{m},(m=$ $1,2, \ldots)$ are obtained recursively by solving the linear part of the equation that results from substituting (10) in the governing equations (4) using $F_{0}(\eta)$ as an initial approximation. The linearisation technique is based on the assumption that $F_{i}$ becomes increasingly smaller as $i$ becomes large; that is,

$$
\lim _{i \rightarrow \infty} F_{i}=0 .
$$

The initial approximation $F_{0}(\eta)$ must be chosen in such a way that it satisfies the boundary conditions (5) and (6). An appropriate initial guess is

$$
F_{0}(\eta)=\left(\frac{e}{2}+\frac{3}{2}\right) \eta-\left(\frac{3 e}{2}+\frac{1}{2}\right) \eta^{3}+e \eta^{4}
$$

where $e$ is an arbitrary constant which when varied results in multiple solutions. Substituting (10) in the governing equations and neglecting nonlinear terms in $F_{i}, F_{i}^{\prime}, F_{i}^{\prime \prime}$, and $F_{i}^{\prime \prime \prime}$ give

$$
F_{i}^{i v}+a_{1} F_{i}^{\prime \prime \prime}+a_{2} F_{i}^{\prime \prime}+a_{3} F_{i}^{\prime}+a_{4} F_{i}=r_{i-1},
$$


where

$$
\begin{gathered}
a_{1}=R\left(\sum_{m=0}^{i-1} F_{m}-\eta\right), \quad a_{2}=-R\left(3+\sum_{m=0}^{i-1} F_{m}^{\prime}\right), \\
a_{3}=-R \sum_{m=0}^{i-1} F_{m}^{\prime \prime}, \quad a_{4}=R \sum_{m=0}^{i-1} F_{m}^{\prime \prime \prime}, \\
r_{i-1}=-\sum_{m=0}^{i-1} F_{m}^{i v}+R\left(\sum_{m=0}^{i-1} F_{m}^{\prime} \sum_{m=0}^{i-1} F_{m}^{\prime \prime}-\sum_{m=0}^{i-1} F_{m} \sum_{m=0}^{i-1} F_{m}^{\prime \prime \prime}\right. \\
\left.+\eta \sum_{m=0}^{i-1} F_{m}^{\prime \prime \prime}+3 \sum_{m=0}^{i-1} F_{m}^{\prime \prime}\right) .
\end{gathered}
$$

Starting from the initial approximation, $F_{0}$, the subsequent solutions for $F_{m}, m \geq 1$, are obtained iteratively by solving (13) subject the the boundary conditions

$$
\begin{aligned}
& F_{i}^{\prime}=0, \quad F_{i}=0, \quad \text { on } \eta=1, \\
& F_{i}^{\prime \prime}=0, \quad F_{i}=0, \quad \text { on } \eta=0 .
\end{aligned}
$$

Once each solution for $F_{i}(i \geq 1)$ has been obtained, the approximate solutions for $F(\eta)$ are obtained as

$$
F(\eta) \approx \sum_{m=0}^{M} F_{m}(\eta),
$$

where $M$ is the order of SLM approximation. It is worth noting that the coefficient parameters and the right hand side of (13) for $i=1,2,3, \ldots$, are known (from previous iterations). Thus, system (13) can easily be solved using numerical methods such as finite differences, finite elements, Runge-Kutta based shooting methods, or collocation methods. In this work, (13) is solved using the Chebyshev spectral collocation method. This method is based on approximating the unknown functions by the Chebyshev interpolating polynomials in such a way that they are collocated at the Gauss-Lobatto points defined as

$$
z_{j}=\cos \frac{\pi j}{N}, \quad j=0,1, \ldots, N,
$$

where $N$ is the number of collocation points used (see e.g., $[20,21])$. In order to implement the method, the physical region $[0,1]$ is transformed into the region $[-1,1]$ using the mapping

$$
\eta=\frac{z+1}{2}, \quad-1 \leq z \leq 1 .
$$

The derivative of $F_{i}$ at the collocation points is represented as

$$
\frac{d^{s} F_{i}}{d \eta^{s}}=\sum_{k=0}^{N} \mathbf{D}_{k j}^{s} F_{i}\left(z_{k}\right), \quad j=0,1, \ldots, N,
$$

where $\mathbf{D}=((2 / L) \mathscr{D})^{s}$ and $\mathscr{D}$ is the Chebyshev spectral differentiation matrix (see, e.g., [20, 21]). Substituting (17)(19) in (13) results in the matrix equation

$$
\mathbf{A}_{i-1} \mathbf{F}_{i}=\mathbf{R}_{i-1}
$$

TABLE 1: The tenth order SLM approximation for $F^{\prime}(0)$ and $F^{\prime \prime}(1)$ at the two branches of solutions for different values of $R$.

\begin{tabular}{lcccc}
\hline & \multicolumn{2}{c}{ Lower branch } & \multicolumn{2}{c}{ Upper branch } \\
$R$ & SLM & bvp4c & SLM & bvp4c \\
\hline \multicolumn{5}{c}{$F^{\prime}(0)$} \\
-3.45 & 3.39748 & 3.39748 & 5.45425 & 5.45425 \\
-3.40 & 3.12576 & 3.12576 & 6.27172 & 6.27172 \\
-3.35 & 2.94758 & 2.94758 & 7.05936 & 7.05936 \\
-3.30 & 2.81355 & 2.81355 & 7.88138 & 7.88138 \\
-3.25 & 2.70596 & 2.70596 & 8.77609 & 8.77609 \\
\hline & \multicolumn{5}{c}{$F^{\prime \prime}(1)$} \\
-3.45 & 7.28689 & 7.28689 & 19.95709 & 19.95709 \\
-3.40 & 5.80900 & 5.80900 & 25.70501 & 25.70502 \\
-3.35 & 4.86274 & 4.86274 & 31.60366 & 31.60366 \\
-3.30 & 4.16178 & 4.16178 & 38.12272 & 38.12272 \\
-3.25 & 3.60511 & 3.60511 & 45.62417 & 45.62417 \\
\hline
\end{tabular}

in which $\mathbf{A}_{i-1}$ is a $(N+1) \times(N+1)$ square matrix and $\mathbf{F}_{i}$ and $\mathbf{R}_{i-1}$ are $(N+1) \times 1$ column vectors defined by

$$
\begin{gathered}
\mathbf{F}_{i}=\left[F_{i}\left(z_{0}\right), F_{i}\left(z_{1}\right), \ldots, F_{i}\left(z_{N-1}\right), F_{i}\left(z_{N}\right)\right]^{T}, \\
\mathbf{R}_{i-1}=\left[r_{i-1}\left(z_{0}\right), r_{i-1}\left(z_{1}\right), \ldots, r_{i-1}\left(z_{N-1}\right), r_{i-1}\left(z_{N}\right)\right]^{T}, \\
\mathbf{A}_{i-1}=\mathbf{D}^{4}+\mathbf{a}_{1} \mathbf{D}^{3}+\mathbf{a}_{2} \mathbf{D}^{2}+\mathbf{a}_{3} \mathbf{D}+\mathbf{a}_{4} .
\end{gathered}
$$

In the above definitions, $\mathbf{a}_{k}(k=1,2,3,4)$ are diagonal matrices of size $(N+1) \times(N+1)$. After modifying the matrix system (20) to incorporate boundary conditions, the solution is obtained as

$$
\mathbf{F}_{i}=\mathbf{A}_{i-1}^{-1} \mathbf{R}_{i-1} \text {. }
$$

\section{Results and Discussion}

In this section, we present the results for the solution of the governing nonlinear boundary value problem (4). To check the accuracy of the successive linearisation method (SLM), comparison is made with numerical solutions obtained using the MATLAB routine bvp4c. The MATLAB routine bvp4c is based on an adaptive Lobatto quadrature scheme [22, 23].

Table 1 gives a comparison between the 10th order SLM approximate results and the bvp4c numerical results for $F^{\prime}(0)$ and $F^{\prime \prime}(1)$ at selected values of $R$. By using different values of the constant $e$ in the initial approximation (see (12)), it was found that both the SLM and bvp4c give multiple solutions when $R<0$. Two solutions, called lower branch and upper branch, were identified when $-3.45 \leq R \leq-3.25$. We observe that the SLM results are in very good agreement with the bvp4c results for both branches of the solutions. Table 1 indicates that the skin friction $F^{\prime \prime}(1)$ decreases when $R$ is increased in the lower branch and the opposite effect is observed when $R$ is increased in the upper branch.

Figure 2 illustrates a slice of bifurcation diagram in both planes. For symmetrical squeezing of the plates; that is, $R>0$, 


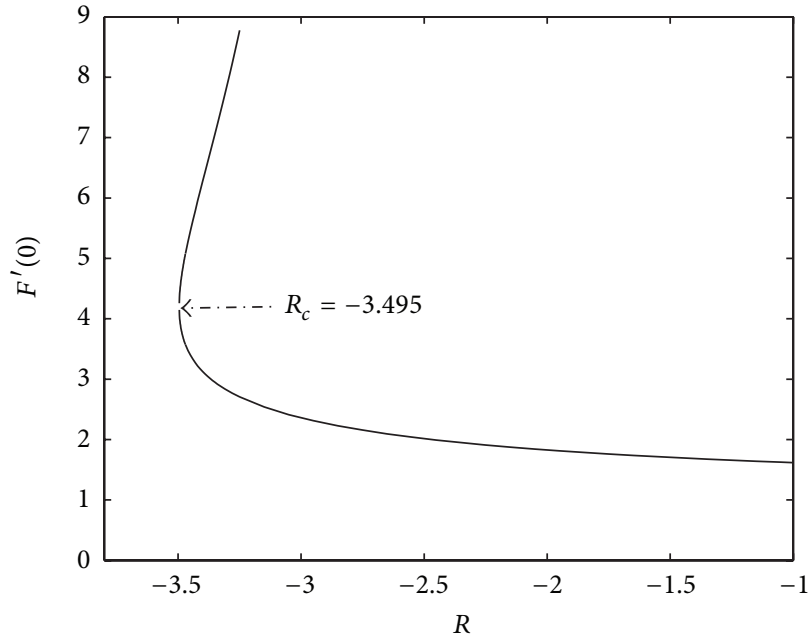

(a)

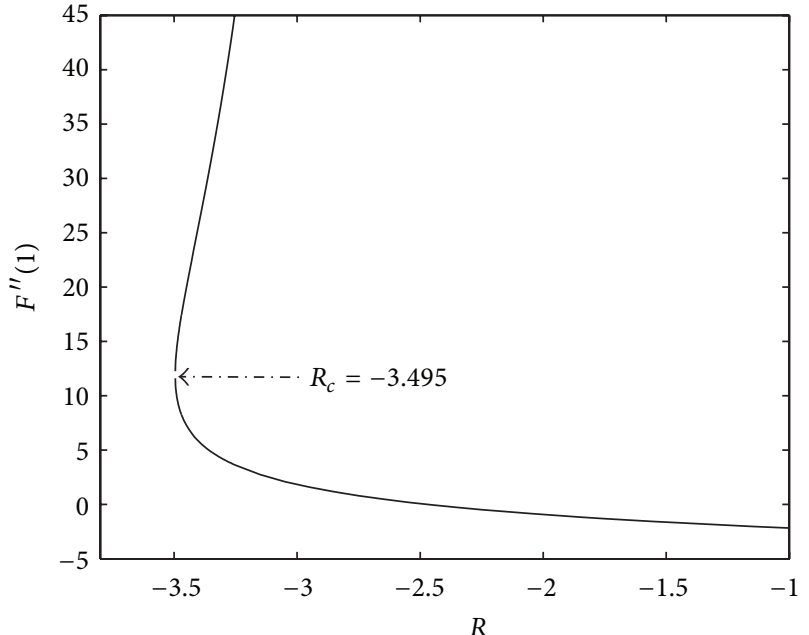

(b)

FIgURE 2: Slice of the bifurcation diagrams for $F^{\prime}(0)$ and $F^{\prime \prime}(1)$ against $R$.

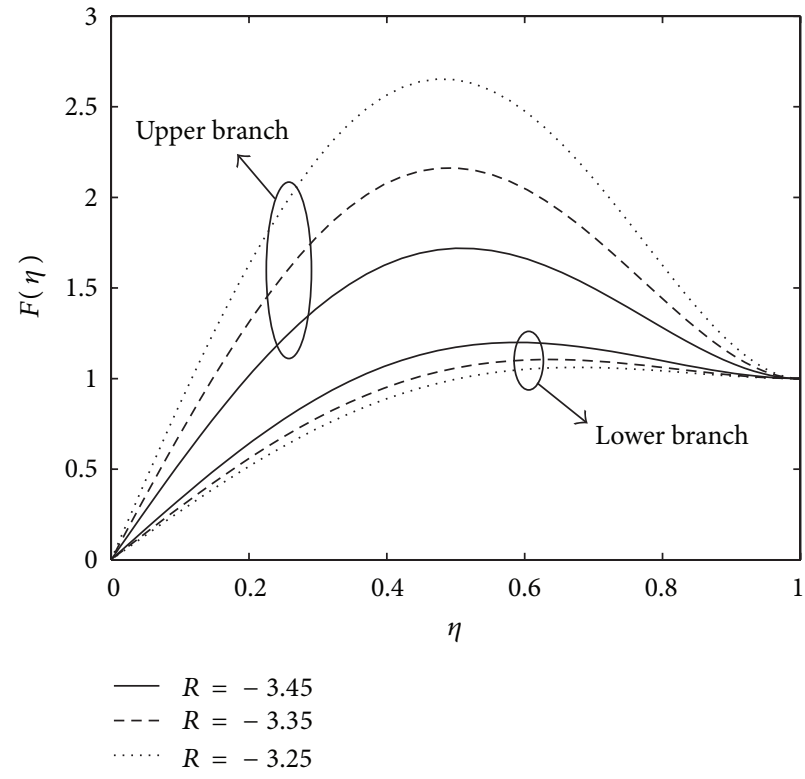

(a)

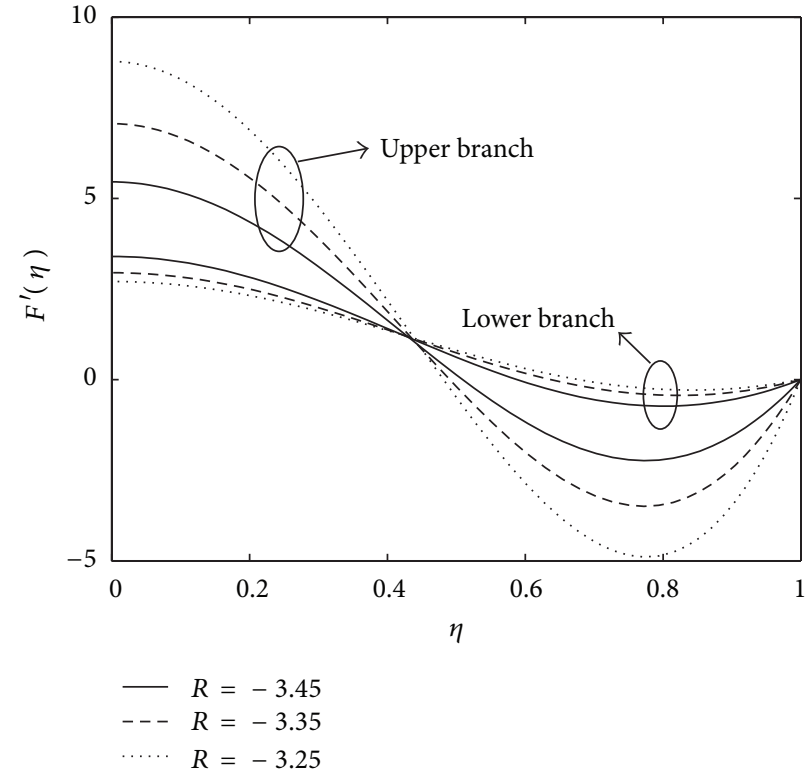

(b)

FIGURE 3: Two branches of the 10th order SLM approximate solution for normal velocity profile $F(\eta)$ and longitudinal velocity profile $F^{\prime}(\eta)$ for different values of $R$.

only one solution branch exists; this can be regarded as the lower solution branch. Another solution branch was identified in addition to the lower solution branch when the plates were symmetrically separated $(R<0)$; this is the upper solution branch. A turning point exists between the primary and secondary solution branches at $R=-3.495$. This bifurcation result obtained using SLM is in perfect agreement with the one reported by Makinde et al. [5] using HermitePadé approximation technique. Moreover, this turning point is very significant with respect to application; it represents the symmetrical separation limit of plates during flow process.
Figure 3 depicts both the fluid normal and axial velocity components during plate separation. It is interesting to note from the lower solution branch that both the fluid normal and axial velocity components increase with an increase in the plates separation (i.e., $R<0$ ) whereas the trend is opposite for upper solution branch. Meanwhile, we observe the possibility of flow reversal near the plates with increasing plate separation. Figure 4 displays the fluid normal and axial velocity components during plate squeezing $(R>0)$ for the only solution branch in this region. Both the normal and axial velocity components decrease with an increase in plates squeezing. 


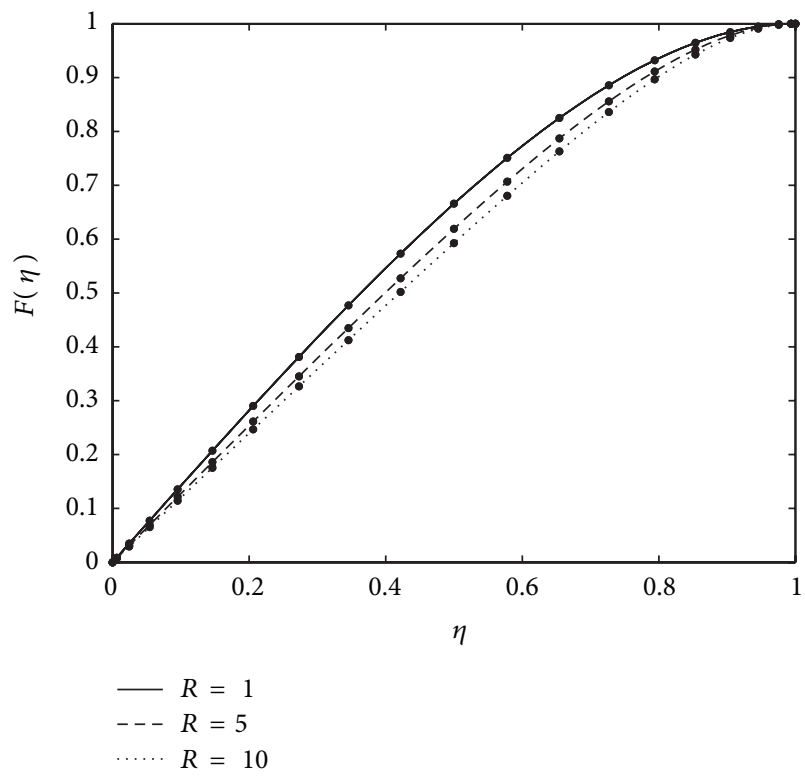

(a)

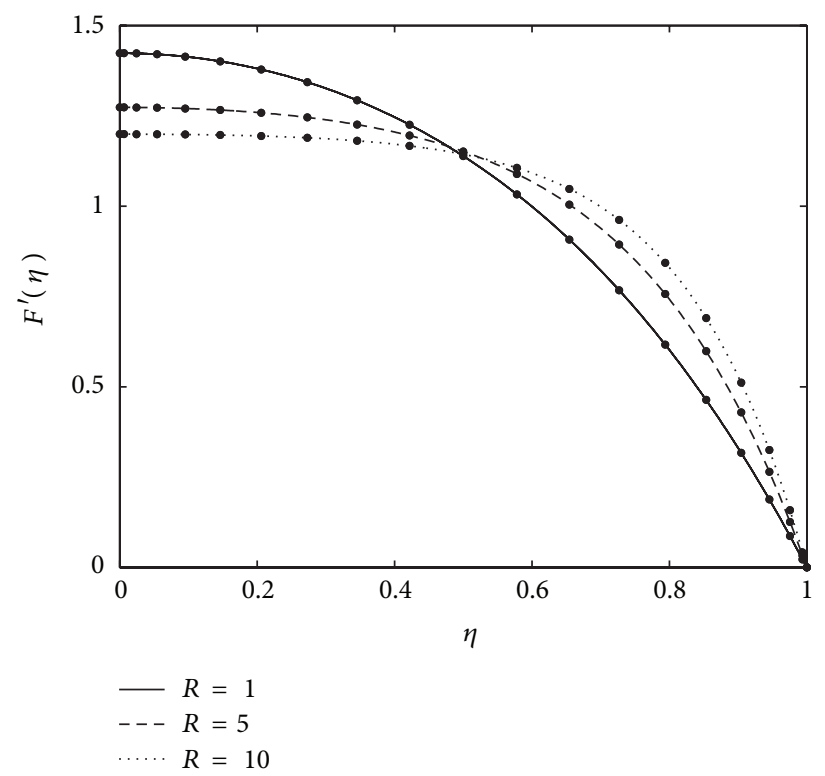

(b)

FIGURE 4: Comparison between the 10th order SLM approximate solution (circles) and the bvp4c numerical results for the normal velocity profile $F(\eta)$ and longitudinal velocity profile $F^{\prime}(\eta)$ for different values of $R$.

\section{Conclusion}

In this work, we employed a very powerful new linearisation technique, known as the successive linearisation method (SLM), to solve a fourth order nonlinear differential equation modelling the transient flow of an incompressible viscous fluid between two parallel plates produced by a simple wall motion. The SLM results for the governing flow parameters were compared with results obtained using MATLAB's bvp4c function and excellent agreement was observed. Using the SLM, it was also shown that the governing problem admits multiple solutions when $R<0$. The ability of the SLM to generate multiple solutions makes it superior to most numerical methods which are only capable of generating one solution of nonlinear equations. Another significant advantage of the SLM is that its implementation does not depend on small parameters unlike other traditional perturbation methods. The study confirms that the proposed SLM approach converges rapidly to the solution of the original nonlinear problem and can be used to solve many other nonlinear equations arising in fluid mechanics and nonlinear science in general.

\section{Conflict of Interests}

The authors declare that there is no conflict of interests.

\section{References}

[1] C. D. Bertram, "Unstable equilibrium behaviour in collapsible tubes," Journal of Biomechanics, vol. 19, no. 1, pp. 61-69, 1986.

[2] J. D. Jackson, "A study of squeezing flow," Applied Scientific Research A, vol. 11, no. 1, pp. 148-152, 1963.

[3] C. D. Bertram and T. J. Pedley, "A mathematical model of unsteady collapsible tube behaviour," Journal of Biomechanics, vol. 15, no. 1, pp. 39-50, 1982.
[4] N. M. Bujurke, P. K. Achar, and N. P. Pai, "Computer extended series for squeezing flow between plates," Fluid Dynamics Research, vol. 16, no. 2-3, pp. 173-187, 1995.

[5] O. D. Makinde, T. G. Motsumi, and M. P. Ramollo, "Squeezing flow between parallel plates: a bifurcation study," Far East Journal of Applied Mathematics, vol. 9, no. 2, pp. 81-94, 2002.

[6] C. Y. Wang, "Squeezing of fluid between two plates" ASME Journal of Applied Mechanics, vol. 43, no. 4, pp. 579-583, 1976.

[7] S. Ishizawa, "The unsteady flow between two parallel discs with arbitrary varying gap width," Bulletin of JSME, vol. 9, no. 35, pp. 533-550, 1966.

[8] O. D. Makinde, "Fluid dynamics of parallel plates viscometer: a case study of methods of series summation," Quaestiones Mathematicae, vol. 26, no. 4, pp. 405-417, 2003.

[9] S. Abbasbandy and T. Hayat, "Solution of the MHD FalknerSkan flow by homotopy analysis method," Communications in Nonlinear Science and Numerical Simulation, vol. 14, no. 9-10, pp. 3591-3598, 2009.

[10] S. Liao, Beyond Perturbation: Introduction to the Homotopy Analysis Method, vol. 2 of Modern Mechanics and Mathematics, Chapman \& Hall/CRC Press, 2003.

[11] S.-J. Liao, "A uniformly valid analytic solution of twodimensional viscous flow over a semi-infinite flat plate," Journal of Fluid Mechanics, vol. 385, pp. 101-128, 1999.

[12] J.-H. He, "Homotopy perturbation technique," Computer Methods in Applied Mechanics and Engineering, vol. 178, no. 3-4, pp. 257-262, 1999.

[13] J.-H. He, "A coupling method of a homotopy technique and a perturbation technique for non-linear problems," International Journal of Non-Linear Mechanics, vol. 35, no. 1, pp. 37-43, 2000.

[14] S. S. Motsa, P. Sibanda, and S. Shateyi, "A new spectralhomotopy analysis method for solving a nonlinear second order BVP," Communications in Nonlinear Science and Numerical Simulation, vol. 15, no. 9, pp. 2293-2302, 2010. 
[15] S. S. Motsa, P. Sibanda, F. G. Awad, and S. Shateyi, "A new spectral-homotopy analysis method for the MHD JefferyHamel problem," Computers \& Fluids, vol. 39, no. 7, pp. 12191225, 2010.

[16] J.-H. He, "Variational iteration method-a kind of non-linear analytical technique: some examples," International Journal of Non-Linear Mechanics, vol. 34, no. 4, pp. 699-708, 1999.

[17] S. S. Motsa and S. Shateyi, "A successive linearization method approach to solve Lane-Emden type of equations," Mathematical Problems in Engineering, vol. 2012, Article ID 280702, 14 pages, 2012.

[18] Z. G. Makukula, P. Sibanda, S. S. Motsa, and S. Shateyi, "On new numerical techniques for the MHD flow past a shrinking sheet with heat and mass transfer in the presence of a chemical reaction," Mathematical Problems in Engineering, vol. 2011, Article ID 489217, 19 pages, 2011.

[19] S. S. Motsa and S. Shateyi, "Successive linearisation analysis of unsteady heat and mass transfer from a stretching surface embedded in a Porous medium with suction/injection and thermal radiation effects," Canadian Journal of Chemical Engineering, vol. 90, no. 5, pp. 1323-1335, 2011.

[20] C. Canuto, M. Y. Hussaini, A. Quarteroni, and T. A. Zang, Spectral Methods in Fluid Dynamics, Springer, Berlin, Germany, 1988.

[21] L. N. Trefethen, Spectral Methods in MATLAB, vol. 10 of Software, Environments, and Tools, SIAM, Philadelphia, Pa, USA, 2000.

[22] J. Kierzenka and L. F. Shampine, "A BVP solver based on residual control and the MATLAB PSE," ACM Transactions on Mathematical Software, vol. 27, no. 3, pp. 299-316, 2001.

[23] L. F. Shampine, I. Gladwell, and S. Thompson, Solving ODEs with MATLAB, Cambridge University Press, Cambridge, UK, 2003. 


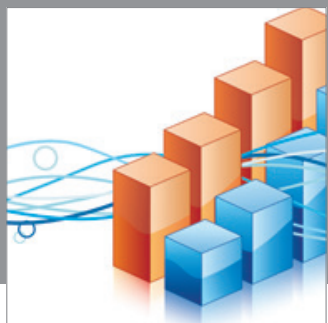

Advances in

Operations Research

mansans

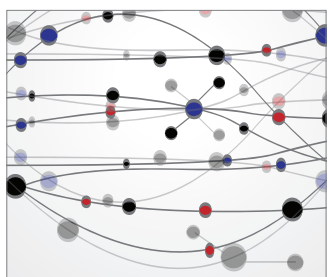

The Scientific World Journal
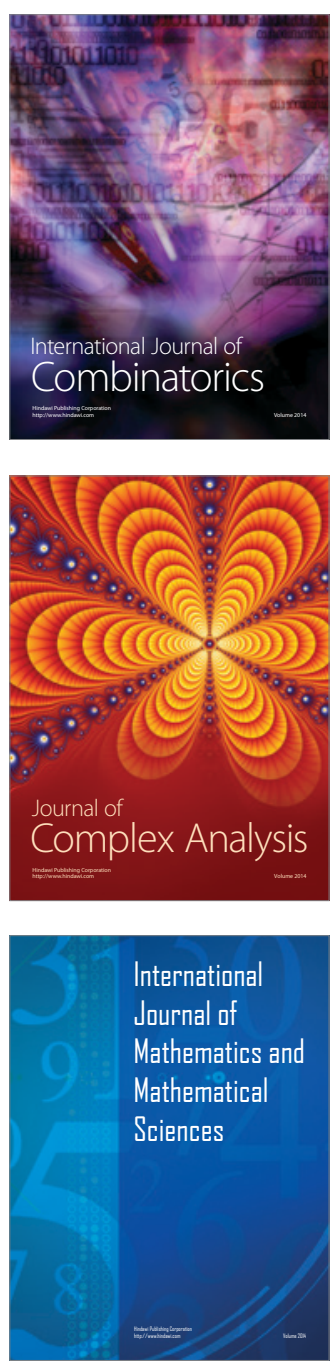
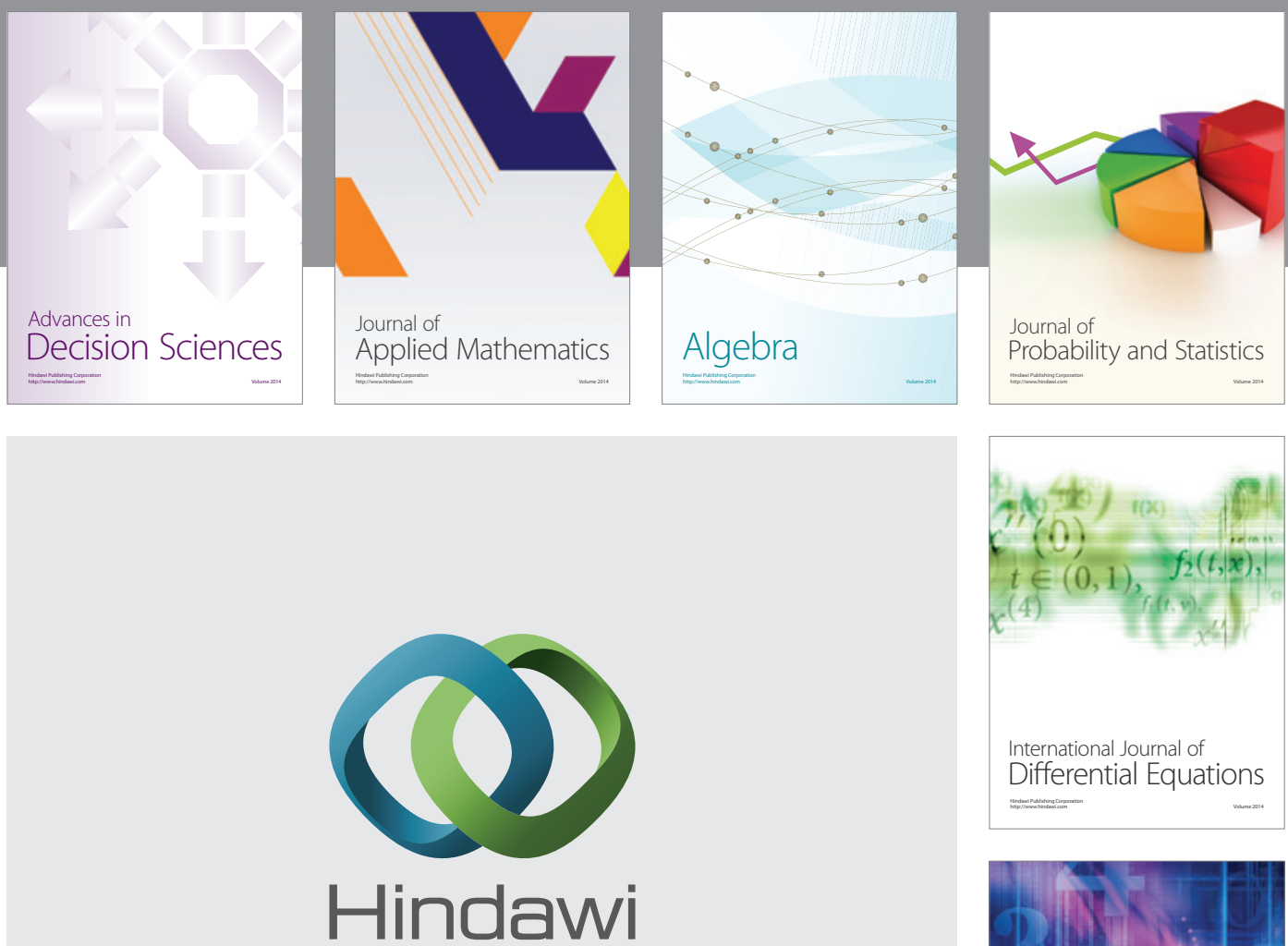

Submit your manuscripts at http://www.hindawi.com
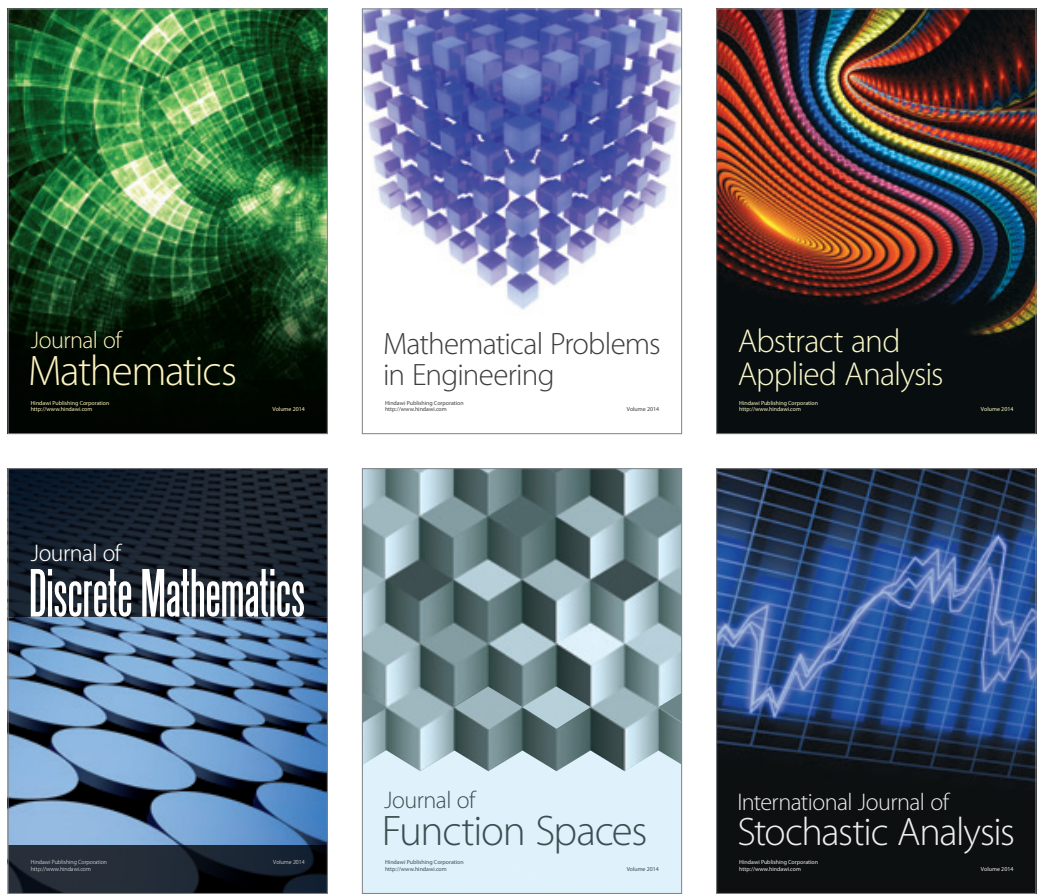

Journal of

Function Spaces

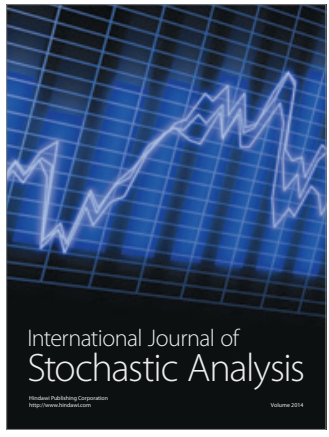

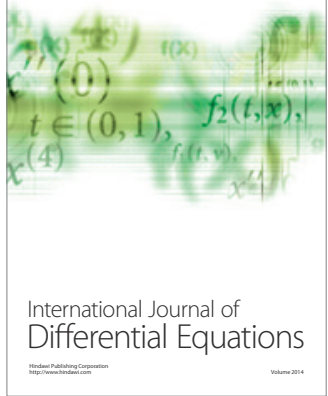
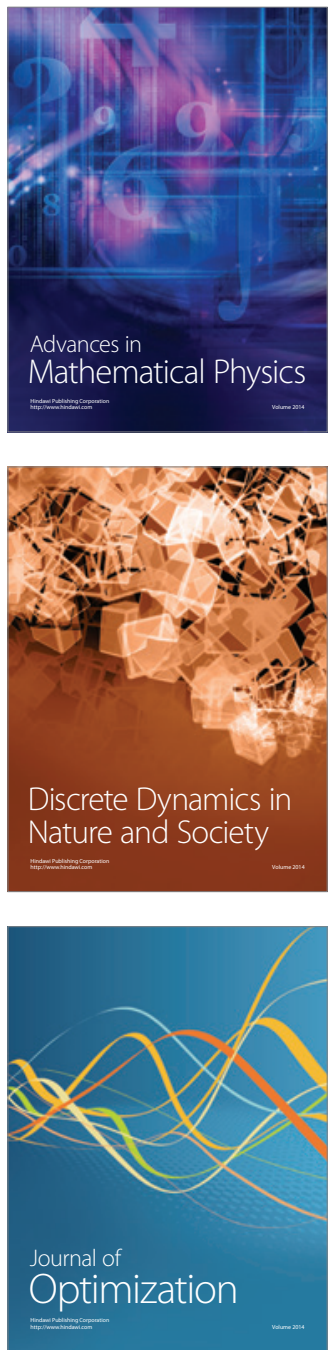